\title{
MÁS ALLÁ DEL CAMBIO CONCEPTUAL: EL APRENDIZAJE DE LA CIENCIA COMO CAMBIO REPRESENTACIONAL
}

\author{
POZO, JUAN IGNACIO \\ Departamento de Psicología Básica. Facultad de Psicología. Universidad Autónoma de Madrid. \\ 28049 Madrid. E-mail: nacho.pozo@uam.es
}

\section{SUMMARY}

In this article we present some reflections on the cognitive changes that should happen in the students' minds when they learn science, as well as on the didactic strategies that could foster such changes.

\section{LOS ECOS DE UNA VIEJA PREGUNTA}

Hace ya unos cuantos años, más en realidad de los que me gustaría, tuve ocasión de leer y discutir en un seminario que por entonces teníamos lo que aún era un preprint de un capítulo de Susan Carey (1985b) finalmente publicado con el título «Are children fundamentally different kinds of thinkers and learners than adults?», que podría traducirse de modo aproximado como « ¿Son los niños fundamentalmente diferentes de los adultos como pensadores y aprendices?». El artículo suponía entonces un reto radical a las posiciones piagetianas en psicología evolutiva al defender una continuidad natural entre los niños y los adultos como sistemas cognitivos, ya que, según defendía la autora, no había en el desarrollo un cambio esencial en el formato representacional del conocimiento, sino únicamente en los contenidos de esas representaciones. En el tiempo transcurrido, en el que incluso las ideas de esta autora han evolucionado hacia posiciones menos continuistas (Carey, 1995), esta pregunta, con un contenido e incluso un «formato representacional» distinto, me ha venido a la mente en innumerables ocasiones, hasta convertirse casi en una obsesión e incluso en la guía de muchas investigaciones emprendidas. En una de sus versiones más habituales, en relación con el aprendizaje de la ciencia, esta pregunta me la formulo en los siguientes términos: «¿Son los alumnos fundamentalmente diferentes de los científicos como pensadores y aprendices?». O, en otras palabras, debemos preguntarnos qué cambios cognitivos, en los procesos y formatos representacionales, tienen que producirse en los alumnos para que puedan utilizar eficazmente los conocimientos científicos que con tanto sudor y lágrimas, y con tan poco éxito, se les enseñan.

Viene a cuento el recuerdo de esta obsesión por la reciente publicación en las páginas de esta revista de un sugerente debate sobre el cambio conceptual (Marín, 1999a, 1999b; Oliva, 1999a, 1999b), que me ha hecho revivir una vez más estos fantasmas cognitivos y al mismo tiempo percibir cómo han ido evolucionando, de forma no siempre explícita o manifiesta, nuestros esfuerzos por responder a estas preguntas, que el mencionado debate no sólo evoca sino que reformula en aspectos esenciales. En las próximas páginas presentaré algunas reflexiones que, desde la psicología cognitiva del apren- 
dizaje, pueden ayudar no a dar respuesta a esa pregunta - ¿de qué viviríamos entonces?, ¿cuáles serían nuestras obsesiones?-, pero sí a buscar caminos que conduzcan a ella. Por lo tanto, centraré mis argumentos en los cambios cognitivos que deberían tener lugar en la mente del alumno cuando aprende ciencia y sólo al final, y de modo muy breve me referiré a las estrategias didácticas que podrían, desde mi punto de vista, favorecer dichos cambios.

\section{¿ES CONCEPTUAL EL CAMBIO CONCEP- TUAL?}

Un primer problema suscitado por Marín (1999a) es por qué llamar a estas alturas cambio conceptual a lo que sin duda es algo más que un cambio de conceptos, algo en lo que también concuerda Oliva (1999a). Como sugiere el título de este trabajo, el verdadero cambio conceptual -la reestructuración fuerte en el sentido de Carey (1985a) - debería implicar no sólo la sustitución o modificación radical de los conceptos o ideas de los alumnos sobre los fenómenos que estudia la ciencia, sino sobre todo un cambio en la forma de concebirlos o, como ha dicho White (1994), un cambio concepcional más que conceptual. Ligándolo a la vieja pregunta formulada por Carey (1985b), el cambio conceptual implicaría un cambio en los procesos y representaciones mediante los que los alumnos procesan los fenómenos científicos y no sólo un cambio en el contenido de esas representaciones.

¿Pero qué tipo de cambio representacional? La respuesta no es fácil, porque, como he sostenido en otras ocasiones (Pozo, 1993; Pozo et al., 1991), las investigaciones en este ámbito no han sido demasiado rigurosas al analizar la naturaleza representacional de las concepciones alternativas -o como se dieran en llamar en cada estudio- que encontraban. Así, se dispone ya de catálogos bastante exhaustivos sobre el contenido de esas representaciones (Driver et al., 1994), pero se ha reflexionado mucho menos sobre en qué consisten esas representaciones y qué tiene que cambiar en ellas para acercarse a los modelos científicos. Si algo tienen en común los muy diversos modelos teóricos que se sitúan dentro del enfoque cognitivo es interpretar la conducta, las acciones y las representaciones de las personas en términos de un sistema de representaciones y procesos cuya naturaleza, sin embargo, varía considerablemente de un modelo o teoría a otro. Aprender ciencia es también cambiar el tipo de procesos y representaciones desde los que se abordan los problemas y situaciones a los que nos enfrentamos.

Dejando a un lado otras muchas diferenciaciones representacionales, una distinción relevante para el aprendizaje de la ciencia es la que hace referencia a la naturaleza implícita/explícita de esos procesos y representaciones (Pozo, 1996, 1998). Las concepciones alternativas de los alumnos pueden interpretarse en el marco de sus teorías implícitas, es decir, como representaciones implícitas generadas por procesos cognitivos implícitos, basados en reglas de carácter esencialmente asociativo e induc- tivo. Los alumnos, como todos nosotros, adquieren representaciones sobre el mundo que les permiten detectar sus regularidades, haciéndolo más predecible y controlable. Pero todo esto sucede de forma implícita, o sea, sin que la persona sea consciente ni de los procesos que utiliza para hacer esas predicciones o acciones, ni en muchos casos de sus propias representaciones, si no es a un nivel muy superficial (esto lo hemos argumentado con más detalle en Pozo y Gómez Crespo, 1998).

De esta forma los alumnos tienen un conocimiento implícito que les proporciona «reglas prácticas», similares a las que se suponía que constituían la esencia del conocimiento científico en los modelos inductivos de ciencia (Dunbar, 1995). Pero si quieren aprender la ciencia que se les enseña deben utilizar procesos explícitos o deliberados para generar representaciones igualmente explícitas, con lo que el proceso de cambio conceptual requeriría una explicitación progresiva de esas representaciones y procesos (Pozo y Gómez Crespo, 1998). De hecho, parece haber un consenso creciente sobre el carácter metacognitivo o, si se prefiere, metaconceptual (Pozo, Gómez Crespo y Sanz, 1999; Vosniadou, 1994) del cambio conceptual y, más en general, del aprendizaje de la ciencia (Campanario y Moya, 1999; Sanmartí, Jorba e Ibáñez, 1999; White, 1999). En mi opinión, ese carácter metacognitivo debería analizarse a lo largo del continuo cognición implícita/explícita, ya que no cabe duda a estas alturas de que buena parte de la metacognición es en realidad implícita (Martí, 1999; Pozo, 1998; Redore, 1996; Schraw y Moshman, 1995).

Una versión interesante de este continuo de explicitación es la de Karmiloff-Smith (1992), según la cual se trataría de un proceso de redescripción representacional. Las nuevas representaciones, los nuevos modelos, deberían ser capaces de redescribir los anteriores y de esta forma explicitarlos o explicarlos. Pero KarmiloffSmith (1992) utiliza el concepto de explicitación en un doble sentido, muy pertinente a efectos de las ambigüedades conceptuales detectadas por Marín (1999a) y Oliva (1999a) en sus críticas al cambio conceptual. Cuando afirmamos que los alumnos tienen conocimientos implícitos, podemos hacerlo en dos sentidos distintos, que no tienen por qué coincidir en una misma representación (Pozo, 1998). En primer lugar, pueden ser implícitos porque el sujeto no puede dar cuenta de ellos, porque no son conscientes o explicitables. Pero también pueden ser implícitos en un sentido más profundo porque ni siquiera están explícitamente presentes en la memoria permanente del alumno. En otras palabras, las concepciones de los alumnos suelen ser implícitas en el sentido de que ni siquiera están presentes en su memoria, listas para ser activadas, sino que son verdaderas construcciones situacionales. Como señala Oliva (1999a), las concepciones alternativas de los alumnos, en cuanto parte de sus teorías implícitas, se corresponden más con modelos mentales que con representaciones esquemáticas, explícitamente presentes en la memoria del sujeto (Moreira, 1996; Rodrigo, 1997; Rodrigo y Correa, 1999). Sin embargo, gran parte de la investigación sobre las ideas de los alumnos suele asumir -¿implícitamente?-que los alumnos «tienen» esas ideas 


\section{DEBATES}

Tabla I

Tres dimensiones de cambio en el aprendizaje de la ciencia, tomado de Pozo y Gómez Crespo (1998).

\begin{tabular}{|c|c|c|}
\hline \multicolumn{3}{|c|}{ PRINCIPIOS EPISTEMOLÓGICOS } \\
\hline REALIS MO INGENUO & REALIS MO INTERPRET ATIVO & CONSTRUCTIVISMO \\
\hline $\begin{array}{l}\text { La realidad es tal como la vemos. } \\
\text { Lo que no se percibe no se concibe. }\end{array}$ & $\begin{array}{l}\text { La realidad existey tiene sus propiedades, } \\
\text { aunque no siempre podamos conocerla } \\
\text { directamente, pero mediante la ciencia } \\
\text { y la técnica podemos saber cómo es } \\
\text { realmente. }\end{array}$ & $\begin{array}{l}\text { El conocimiento científico es } \\
\text { una construcción que nos proporciona } \\
\text { modelos alternativos para interpretar } \\
\text { la realidad, pero que no son parte } \\
\text { de la realidad. }\end{array}$ \\
\hline & PRINCIPIOS ONTOLÓGICOS & \\
\hline ES TADOS & PROCESOS & SISTEMAS \\
\hline $\begin{array}{l}\text { Interpretación del mundo en términos } \\
\text { de estados de la materia desconectados } \\
\text { entre sí. }\end{array}$ & $\begin{array}{l}\text { Los fenómenos se interpretan como una } \\
\text { sucesión de hechos relacionados entre sí } \\
\text { mediante ciertos procesos. }\end{array}$ & $\begin{array}{l}\text { Los fenómenos se interpretan a partir } \\
\text { del conjunto de relaciones complejas } \\
\text { que forman parte de un sistema. }\end{array}$ \\
\hline & PRINCIPIOS CONCEPTUALES & \\
\hline HECHOS O DATOS & $\begin{array}{l}\text { CAUSALIDAD LINEAL } \\
\text { (de simple a múltiple) }\end{array}$ & INTERACCIÓN \\
\hline $\begin{array}{l}\text { Los fenómenos y hechos se describen } \\
\text { en función de las propiedades y cambios } \\
\text { observables. }\end{array}$ & $\begin{array}{l}\text { Los fenómenos se explican mediante } \\
\text { relaciones causales simples } \\
\text { que evolucionan a distintos grados } \\
\text { de complejidad. }\end{array}$ & $\begin{array}{l}\text { Las propiedades de los cuerpos } \\
\text { y los fenómenos se interpretan como } \\
\text { un sistema de relaciones de interacción. }\end{array}$ \\
\hline CAMBIO SIN CONSERVAC IÓN & CAMBIO CON CONSERVACIÓN & CONSERVAC IÓN Y EQUILIBRIO \\
\hline $\begin{array}{l}\text { Sólo cambia aquéllo que vemos que se } \\
\text { altera. Necesidad de explicar lo que } \\
\text { cambia pero no lo que permanece. }\end{array}$ & $\begin{array}{l}\text { Se aceptala conservación de propiedades } \\
\text { no observables pero no el equilibrio. }\end{array}$ & $\begin{array}{l}\text { Los distintos fenómenos se interpretan } \\
\text { en términos de interacción, lo que lleva } \\
\text { a la conservación y al equilibrio. }\end{array}$ \\
\hline RELAC IONES CUALITATIVAS & REGLAS HEURÍSTICAS & RELAC IONES CUANTITATIVAS \\
\hline $\begin{array}{l}\text { Los fenómenos se interpretan en forma } \\
\text { cualitativa. }\end{array}$ & Utilización de reglas simplificadoras. & Proporción, probabilidad, correlación. \\
\hline
\end{tabular}

de modo permanente. ¿Acaso no era la persistencia y permanencia de esas ideas uno de sus rasgos identificativos? Coincido con Oliva (1999a) en que lo que permanece son los trazos o rasgos estructurales de esos modelos mentales o, si se prefiere, la estructura de las teorías implícitas en las que se basan esas construcciones situacionales que llamamos modelos mentales (Rodrigo, 1997; Rodrigo y Correa, 1999). Además, la interpretación en términos de modelos mentales ayuda a entender mejor los efectos del contexto señalados por Oliva (1999a) y la consistencia variable de esas ideas (Pozo y Gómez Crespo, en prensa).

Así, las concepciones alternativas de los alumnos son en buena medida representaciones implícitas en el sentido de que ni siquiera «están» en su sistema de memoria sino que se construyen situacionalmente a partir de ciertas unidades de información y ciertas estructuras asociati- 
vas que constituyen el «pegamento» de esas ideas. Sin embargo, algunas de esas representaciones, por su construcción o activación repetida en un contexto similar, acabarán por explicitarse o empaquetarse como estructuras de información organizadas, como representaciones esquemáticas, que quizás fuera más acertado llamar representaciones estables en lugar de explícitas. Pero estos esquemas puede que no sean explícitos aún en el segundo sentido, en el de ser accesibles a la conciencia del sujeto. En nuestras investigaciones recientes sobre las concepciones implícitas de profesores y alumnos sobre el aprendizaje y la enseñanza hemos encontrado que no ya los alumnos sino los propios profesores tienen representaciones estables que, sin embargo, no llegan a explicitar sino en sus aspectos más superficiales (Pozo et al., 1999). Lo mismo sucede sin duda con algunas de las concepciones de los alumnos sobre la ciencia: no son conscientes (el alumno no nos dice que es aristotélico o enuncia el principio de atribuir propiedades macroscópicas a las partículas), pero la estabilidad de sus representaciones a través de contextos y tareas diferentes nos indica que dispone de esquemas o representaciones estables para esos contextos (Pozo y Gómez Crespo, 1997).

Para que esa explicitación, entendida como redescripción representacional, es decir, como traducción de una representación a formatos y representaciones más potentes y explícitos, pueda producirse, se requiere en mi opinión disponer de lenguajes o códigos que hagan posible esa nueva representación, pero también de estructuras conceptuales que puedan asimilar los nuevos modelos así representados (Pozo y Gómez Crespo, 1998). Los modelos y teorías científicas que el alumno debe aprender vienen formulados en lenguajes que se alejan bastante de los códigos en los que habitualmente el alumno se representa sus propios conocimientos implícitos, escasamente formalizados, al consistir en acciones, percepciones y concepciones fragmentarias o aisladas. Un aspecto que ha estado muy ausente de la investigación sobre las concepciones de los alumnos y el cambio conceptual ha sido la importancia de los códigos y lenguajes en los que se formulan los modelos científicos. La argumentación como forma de explicitación (Duchsl, 1998; Jiménez Aleixandre, 1998; Ogborn et al., 1996) requiere un cambio representacional en el sentido de redescribir los modelos anteriores en nuevos lenguajes o formatos representacionales (Mortimer, 1998, en prensa). La ciencia es también una forma de hablar o contar las cosas que suceden. No creo, como afirma Marín (1999b), que esto implique dar «demasiada credibilidad al constructivismo social», sino más bien que, en último extremo, para promover estos u otros cambios hay que generar ciertas formas de organización social de las actividades de aprendizaje y enseñanza que como tales, requerirán de soportes o códigos comunicativos que los alumnos, en el mejor de los casos, acabarán por interiorizar.

Pero esa redescripción representacional de las viejas concepciones en las nuevas se ve dificultada también porque las teorías científicas difieren de las teorías implícitas no sólo en su contenido factual, sino también en una serie de principios organizadores, en una serie de supuestos epistemológicos, ontológicos y conceptuales (Pozo y Gómez Crespo, 1998). En la tabla I se proponen algunos principios que mostrarían que el aprendizaje de la ciencia supone evolucionar desde posiciones más o menos realistas (el conocimiento tiende a reflejar la realidad, y el conocimiento científico la refleja mejor que el intuitivo) a posiciones más constructivistas (todo conocimiento es un modelo o aproximación relativo a unas determinadas condiciones de activación o aplicación), pero también evolucionar en complejidad, desde una visión estática a una visión sistémica de los problemas y desde un análisis causal lineal, centrado en los cambios situacionales, a la interpretación mediante esquemas de interacción y equilibrio. En la medida en que esos principios implican una mayor complejidad no se puede redescribir lo más simple en lo más complejo si no se aprenden o construyen esas nuevas estructuras conceptuales. Es la vieja paradoja del aprendizaje: ¿Cómo es posible generar a partir de un sistema de conocimiento más simple otro más complejo que asimile o integre al anterior? Sólo parece posible, de modo generalizado a través de una instrucción específicamente diseñada, pero no de una instrucción directa. No se trata de enseñar a los alumnos esos esquemas o principios generales, sino de ayudarles a construir esos esquemas en dominios o contextos específicos, como posible respuesta a determinados problemas, para que luego se generalice o transfiera a otros nuevos. En este caso, como en otros, los contenidos escolares no deberían ser un fin en sí mismos sino una vía para desarrollar en los alumnos capacidades (Pozo, 1999a), que serían esos principios que, según la tabla I, diferencian las teorías implícitas de las científicas. Pero esto nos lleva a otro problema del cambio conceptual, relativo no ya a su naturaleza conceptual o representacional sino al carácter de ese cambio y a los procesos implicados en él.

\section{¿HAY CAMBIO EN EL CAMBIO CON- CEPTUAL?}

Parece haber un acuerdo creciente, que comparten tanto Marín (1999b) como Oliva (1999a, 1999b) en que el cambio conceptual no puede ni debe implicar la sustitución de un tipo de representación por otro. Igualmente se acepta el carácter complejo, o pluriprocesual, del cambio conceptual. Ambos acuerdos, que también comparto, son muy importantes y deberían tener consecuencias sobre la investigación -y en último extremo sobre la enseñanza-que por ahora no tienen (Pozo, 1994). Empezando por este segundo, la existencia de diversos procesos de complejidad creciente dentro del llamado cambio conceptual -desde el crecimiento y el ajuste para llegar a la reestructuración-debe exigirnos una visión integradora -no una mezcla como apunta Marín (1999b)- del aprendizaje y la enseñanza, de forma que la ya rancia etiqueta del constructivismo dé paso a formas diferentes de concebir esa construcción. Sin duda, a fuerza de enseñar el constructivismo en el marco de formatos didácticos bastante tradicionales, es más fácil encontrar discursos constructivistas que prácticas constructivas 
entre los profesores (Pérez Echeverría et al., en prensa). Muchas de esas prácticas docentes tradicionales son redescribibles, sin embargo - por utilizar la terminología aquí empleada- en el marco de prácticas constructivas. Desde nuestro punto de vista, que no podemos desarrollar aquí con más detalle (Pozo y Gómez Crespo, 1998), el cambio conceptual, o representacional, debería ser la meta pero no el método de esa construcción, la cual debería integrar formas de aprendizaje y enseñanza diferentes. Pero ese cambio en las metas, más que sólo en los métodos, exige de hecho un cambio conceptual, o representacional, por parte de los profesores en su concepción del aprendizaje y la enseñanza, que es mucho más difícil que lograr un cambio técnico, en los recursos didácticos, y que estamos aún muy lejos de alcanzar (Pérez Echeverría et al., en prensa; Porlán, Rivero y Martín del Pozo, 1998; Pozo et al., 1999).

En cuanto al otro consenso logrado en torno a los procesos del cambio conceptual -según el cual no se trata de una sustitución de unas representaciones por otras- no deja de ser un acuerdo en negativo. Se está de acuerdo en lo que no es el cambio conceptual, pero, ¿entonces, qué es? No resulta claro a la luz de los recientes desarrollos en este ámbito. De hecho, creemos que este acuerdo negativo puede tener un cierto efecto paralizante o incluso una vuelta a posiciones que parecían ya superadas. Por nuestra parte, en estas mismas páginas presentamos otro trabajo (Pozo, 1999b) en el que intentábamos enunciar diferentes alternativas, decantándonos finalmente por la integración jerárquica entre diversas formas de conocimiento. La imposibilidad, e incluso la inconveniencia, de sustituir o anular las teorías implícitas de los alumnos -con su larga historia filogenética, ontogenética y cultural detrás- por las teorías o modelos elaborados por los científicos está conduciendo, por un lado, a una recuperación de los discursos sobre la compatibilidad o continuidad entre el conocimiento cotidiano y el científico y, por otro, a un riesgo de excesiva contextualización o relativización del conocimiento científico, que haría muy dudoso el esfuerzo de enseñarlo.

En cuanto a la primera reacción, vendría a sostener que la ciencia es una continuación sofisticada de la forma en que las personas, niños incluidos (Gopnik y Meltzoff, 1997) e incluso otros animales, según Dunbar (1995), resolvemos cotidianamente nuestros problemas. Ante la pregunta con la que comenzábamos este trabajo, la respuesta categórica sería que no hay diferencias fundamentales entre la mente del alumno y la del científico, porque, de hecho, la ciencia no es sino una continuación natural de la forma en la que las personas, y más en general los seres vivos, exploramos y controlamos nuestro entorno. La vieja metáfora del niño como pequeño científico, tan querida por los piagetianos, da incluso paso a una nueva versión en la que serían los científicos los que utilizarían las mismas representaciones y procesos que los niños (Gopnik y Meltzoff, 1997). Así, los programas de la ciencia, su métodos y modelos, corren perfectamente en la mente del alumno, sólo hay que cargarlos (Pozo, 1999b). Por lo tanto, el cambio conceptual, entendido como una reestructuración radical, no es necesario. Aprender ciencia es, de nuevo, una tarea meramente acumulativa, que requiere como máximo unos ciertos ajustes.

En el otro extremo, desde una concepción situada o contextualista del aprendizaje, la no sustitución de las teorías implícitas por las científicas se debería a que son formas de conocer diferentes, que coexisten de modo independiente y sirven para contextos distintos, como muy bien argumenta Oliva (1999a). La paradoja aquí es que, si finalmente los modelos científicos sólo sirven para los contextos científicos, si sólo son eficaces en las condiciones de trabajo intelectual y material de un científico, ¿para qué enseñar ciencia en la educación obligatoria a alumnos que en su inmensa mayoría no van a ser científicos? Una visión radicalmente situada o contextualizada del aprendizaje de la ciencia nos llevaría a negar la posibilidad o necesidad de la transferencia de lo aprendido a nuevas situaciones y contextos, tal como hacen Lave y Wenger (1991; ver también Kirshner y Whiston, 1997), y, por tanto, convertiría en una tarea socialmente inútil el enseñar ciencia a futuros nocientíficos.

Se trata, pues, de conciliar la necesaria coexistencia representacional de las diversas formas científicas e intuitivas de conocimiento con una integración conceptual o jerárquica de las mismas. Frente a una diferenciación contextual, de bajo nivel (si hoy es martes, esto es una clase de química), podemos contraponer una diferenciación metacognitiva, basada en la integración jerárquica entre formas de conocimiento, de manera que las más potentes puedan redescribir o explicar las más simples pero sin que las anulen desde el punto de vista representacional o cognitivo, ya que éstas siguen siendo muy eficaces para asimilar (predecir, controlar, etc.) muchas situaciones cotidianas de modo implícito, en las que poner en marcha un modelo científico de forma explícita sería mucho más costoso en tiempo y recursos cognitivos y, por tanto, menos eficaz. Podemos seguir viendo ponerse el sol y pensando en comprarnos jerseys que den calor sin que eso suponga que ignoremos la posibilidad de redescribir esas situaciones en términos de modelos científicos más precisos pero también más complejos y no más predictivos en esos contextos o escenarios. Se trataría de representaciones diferentes con funciones cognitivas distintas (Pozo, Gómez Crespo y Sanz, 1999)

\section{EL CAMBIO INSTRUCCIONAL FRENTE AL CAMBIO EVOLUTIVO Y EPISTEMO- LÓGICO}

Aunque el propósito de estas páginas sea reflexionar sobre los cambios que tienen lugar en la mente de los alumnos cuando aprenden ciencia, que no siempre es el caso, es inevitable hacer una referencia, aunque sea breve, a las estrategias instruccionales que deben favorecer ese cambio.Uno de los problemas, en mi opinión, es que, al referirse a esa cosa llamada cambio conceptual, suelen darse ciertos saltos argumentales entre niveles distintos de análisis del cambio conceptual: el evolu- 
tivo (los cambios que tienen lugar como consecuencia del desarrollo cognitivo), el epistemológico (los cambios que han tenido lugar en la historia de la ciencia) y el instruccional (los cambios que deben producirse como consecuencia de la enseñanza). No hay motivos para creer que los cambios en los procesos y representaciones tengan que ser los mismos en los tres casos. Durante bastante tiempo, por influencia de la obra piagetiana, se intentó asimilar el cambio instruccional al cambio evolutivo, pero es dudoso que sean comparables (por ejemplo, los procesos de explicitación no pueden jugar el mismo papel en unos y otros; los cambios evolutivos son relativamente «espontáneos» en casi todos los niños; los instruccionales no se producen ni siquiera «a presión»). De un tiempo a esta parte, tiende a asimilarse más bien el cambio instruccional al cambio epistemológico, buscando el paralelismo entre la enseñanza de la ciencia, como contexto de transmisión del conocimiento científico, y la propia investigación científica, como contexto de producción de ese mismo conocimiento. Esta posición ha sido defendida, tanto desde supuestos un tanto inductivistas (Wagensberg, 1993) como claramente constructivistas (Gil et al., 1991). Asumiendo que sin duda los procesos de producción del conocimiento científico y las dificultades que deben superarse en su uso son una fuente esencial de criterios para el diseño de actividades de aprendizaje-enseñanza, hay también bastantes razones para argumentar que el aprendizaje de la ciencia no puede apoyarse, como actividad esencial, en la investigación (si entendemos por tal lo que hacen los científicos), que la actividad de los alumnos aprendiendo ciencia es un contexto diferente al de un científico, y que la actividad de un profesor es muy diferente a la del director de un proyecto de investigación (Pozo y Gómez Crespo, 1998).

Los escenarios escolares tienen una estructura social y cognitiva propia, no reducibles ni a los escenarios de desarrollo ni a las labores de investigación (Coll, 1990, 1993). Si la labor del profesor es en buena medida ayudar al alumno a explicar o redescribir sus propias concepciones implícitas, contrastándolas con otras concepciones explícitas, a través del diseño de ciertos escenarios y actividades de enseñanza, debemos indagar más en las formas de argumentar y contrastar modelos a partir de esas situaciones, en las formas de explicar las ciencias, igual que en otras materias (Ogborn et al.,
1996; Sánchez, 1999). En este sentido, una enseñanza de la ciencia basada en modelos -de los modelos mentales a los científicos- puede hacer compatible el viejo papel de los maestros con las nuevas exigencias de los aprendices como constructores de conocimiento. Si aceptamos, con Giere (1998, 1999), que la ciencia es una determinada forma de construir modelos que representan partes del mundo que conocemos, aprender y enseñar ciencia consistirá, en gran medida en desarrollar, contrastar y argumentar diferentes modelos para diferentes tareas y problemas, de forma que se puedan integrar o redescribir entre sí (Pozo y Gómez Crespo, 1998). No es tanto el contraste empírico en tanto que el contraste teórico el que debe guiar ese proceso de construcción como un proceso de explicitación o, si se quiere, de explicación.

Sin duda, un planteamiento así puede recoger aportaciones de los enfoques de enseñanza de la ciencia como investigación, pero ayuda a recuperar, y también a redescribir la vieja labor del profesor como alguien que explica a sus alumnos lo que sabe, pero en este caso en el marco de una concepción perspectivista (Giere, 1999; Pozo et al., 1999) que le da un nuevo sentido o dimensión, ya que el discurso del profesor no podrá ser nunca unidimensional sino que tendrá que incorporar diferentes voces, o modelos. Por otra parte, la labor del alumno tampoco será ya repetir o recitar lo explicado por el profesor, sino argumentarlo, redescribirlo en función de sus propias teorías implícitas que, con ello, se irán también redescribiendo, explicitando y reestructurando. Pero en cualquier caso, al igual que he sostenido con respecto a las concepciones de los alumnos en relación con los modelos científicos, no se trata tampoco de sustituir una concepción de la enseñanza de la ciencia por otra, sino de buscar la forma de integrar diferentes enfoques. Pero tampoco podemos adoptar una posición relativista o ecléctica según la cual todo vale, dependiendo de la situación, sino que debemos ser capaces, si se permite una vez más, y ya es la última, de elaborar modelos que redescriban eficazmente otras experiencias y modelos, en lugar de buscar el Santo Grial didáctico como a veces se pretende, un método o modelo, cuyo seguimiento conduce inexorablemente al paraíso del aprendizaje. Tampoco la didáctica de la ciencia puede ser unidimensional sino que debe de acoger, y aún más, múltiples voces y modelos, e intentar integrarlos. 


\section{REFERENCIAS BIBLIOGRÁFICAS}

CAMPANARIO, J.M. y MOYA, A. (1999). ¿Cómo enseñar ciencias? Principales tendencias y propuestas. Enseñanza de las ciencias, 17(2), pp. 179-192.

CAREY, S. (1985a). Conceptual change in childhood. MIT: Press: Cambridge, Mass.

CAREY, S. (1985b). Are children fundamentally different kinds of thinkers and learners than adults?, en Chipman, S., Segal, J. y Glaser, R. (eds.). Thinking and learning skills. Vol 2. Hillsdale, N.J.: Erlbaum.

CAREY, S. (1995). On the origin of causal understanding, en Sperber, D. Premack, D. y Premack, A.J. (eds.). Causal cognition. An interdisciplinary debate. Oxford: Oxford Univ. Press.

COLL, C. (1990). Un marco de referencia psicológico para la educación escolar: la concepción constructivista del aprendizaje y la enseñanza, en Coll, C., Palacios, J. y Marchesi, A. (eds.). Psicología de la Educación II. Desarrollo psicológico y educación. Madrid: Alianza.

COLL, C. (1993). Constructivismo e intervención educativa: ¿cómo enseñar lo que se ha de construir?, en Beltrán, J.A., Bermejo, V., Prieto, M.D. y Vence, D. (eds.). Intervención psicopedagógica. Madrid: Pirámide.

DRIVER, R., SQUIRES, A., RUSHWORTH, P. y WOODROBINSON, V. (1994). Making sense of secondary school. Londres: Routledge. Trad. cast. de Pozo, M.J. (1999). Dando sentido a la ciencia en secundaria. Madrid: Visor.

DUCHSL, R.A. (1998). La valoración de argumentaciones y explicaciones: promover estrategias de retroalimentación. Enseñanza de las Ciencias, 16(1), pp. 3-20.

DUNBAR, R. (1995). The trouble with science. Trad. cast. de Ferrero, M. (1999). El miedo a la ciencia. Madrid: Alianza.

GIERE, R.N. (1988). Explaining science: a cognitive approach. Chicago: The University of Chicago Press.

GIERE, R.N.(1999). Unnuevo marcopara enseñar el razonamiento científico. Enseñanza de las Ciencias, núm. extra, pp. 6370 .

GIL, D., CARRASCOSA, J., FURIÓ, C. y MARTÍNEZ TORREGROSA, J. (1991). La enseñanza de las ciencias en la educación secundaria. Barcelona: Horsori.

GOPNIK, A. y MELTZOFF, A.N. (1997). Words, thoughts and theories. Cambridge, Mass.: Cambridge University Press. Trad. cast. de María Sotillo. (1999). Palabras, pensamientos y teorías. Madrid: Visor.

JIMÉNEZ ALEIXANDRE, M.P. (1998). Diseño curricular: indagación y razonamiento con el lenguaje de las ciencias. Enseñanza de las ciencias, 16(2), pp. 203-216.

KARMILOFF-SMITH, A. (1992). Beyond modularity. Cambridge, Mass.: Cambridge University Press. Trad. cast. de Gómez, J.C. y Núñez, M. (1994). Más allá de la modularidad. Madrid: Alianza.

KIRSHNER, D. y WHISTON, J.A. (eds.) (1997). Situated cognition. Social, semiotic and psychological perspectives. Hillsdale, Erlbaum.

LAVE, J. y WENGER, E. (1991). Situated learning. Cambridge, Mass.: Cambridge University Press.

MARÍN, N. (1999a). Delimitando el campo de aplicación del cambio conceptual. Enseñanza de las ciencias, 17(1), pp. 80-92.
MARÍN, N. (1999b). Del cambio conceptual a la adquisición de conocimientos. Enseñanza de las Ciencias, 17(1), pp. 109-114.

MARTÍ,E. (1999). Metacognición y estrategias de aprendizaje, en Pozo, J.I. y Monereo, C. (eds.). El aprendizaje estratégico: enseñar a aprender desde el currículo. Madrid: Santillana.

MOREIRA, M.A. (1996). Modelos mentais. Investigaçoes em ensino de ciências, 1(3), pp. 196-232.

MORTIMER, E. (1998). Multivoicedness and univocality in classroom discourse: an example from theory of matter. International Journal of Science Education, 20(1), pp. 67-82.

MORTIMER, E. (en prensa). Perfiles conceptuales: modos de pensar y modos de hablar en las clases de ciencias. Infancia y Aprendizaje.

OGBORN, J., KRESS, G., MARTINS, I. y McGILLIKUDAY, K. (1996). Explaining science in the classroom. Londres: Open University Press. Trad. cast. De R. Llavori (1998). Formas de explicar. Madrid: Santillana.

OLIVA, J.M. (1999a). Algunas reflexiones sobre las concepciones alternativas y el cambio conceptual._Enseñanza de las Ciencias, 17(1), pp. 93-107.

OLIVA, J.M. (1999b). Ideas para la discusión sobre el cambio conceptual. Enseñanza de las Ciencias, 17(1), pp. 115-117.

PÉREZ ECHEVERRÍA, M.P., MATEOS, M., POZO, J.I. y SCHEUER, N. (en prensa). En busca del constructivismo perdido: las teorías implícitas sobre el aprendizaje y la enseñanza, en Cubero, R. y Luque, A. (eds.). Constructivismo y educación.

PORLÁN, R., RIVERO, A. y MARTÍN DEL POZO, R. (1997). Conocimiento profesional y epistemología de los profesores I: Teoría, métodos e instrumentos. Enseñanza de las Ciencias, 15(2), pp. 155-171.

POZO, J.I. (1993). Psicología y didáctica de las ciencias de la naturaleza: ¿concepciones alternativas? Infanciay Aprendizaje, 62-63, pp. 187-204.

POZO, J.I. (1994). El cambio conceptual en el conocimiento físico y social: del desarrollo a la instrucción, en Rodrigo, M.J. (ed.). Contexto y desarrollo social. Madrid: Síntesis.

POZO, J.I. (1996). Aprendices y maestros. Madrid: Alianza Editorial.

POZO, J.I. (1998). Las relaciones entre conocimiento implícito y conocimiento explícito en el aprendizaje y la instrucción. Seminario sobre Perspectivas Actuales en Psicología Cognitiva. Universidad Autónoma de Madrid. Miraflores de la Sierra, octubre de 1998.

POZO, J.I. (1999a). Aprendizaje de contenidos y desarrollo de capacidades en la educación secundaria, en Coll, C. (ed.). Psicología de la instrucción: la enseñanza y el aprendizaje en la educación secundaria. Barcelona: Horsori.

POZO, J.I. (1999b). Sobre las relaciones entre el conocimiento cotidiano de los alumnos y el conocimiento científico: del cambio conceptual a la integración jerárquica. Enseñanza de las Ciencias, núm extra, pp. 15-29.

POZO, J.I.y GÓMEZ CRESPO, M.A.(1997). Cambio conceptual en química. Memoriade investigación (no publicada). Facultad de Psicología de la Universidad Autónoma de Madrid. 
POZO, J.I. y GÓMEZ CRESPO, M.A. (1998). Aprender y enseñar ciencia. Del conocimiento cotidiano al conocimiento científico. Madrid: Morata.

POZO, J.I. y GÓMEZ CRESPO, M.A. (en prensa). La consistencia de las teorías sobre la naturaleza de la materia. Una comparación entre expertos y novatos. Infancia y Aprendizaje.

POZO, J.I., GÓMEZ CRESPO, M.A., LIMÓN, M. y SANZ, A. (1991). Procesos cognitivos en la comprensión de la ciencia: ideas de los alumnos sobre la química. Madrid: Servicio de Publicaciones del MEC.

POZO, J.I., GÓMEZ CRESPO, M.A. y SANZ, A. (1999). When conceptual change does not mean replacement: different representations for different contexts, en Schnotz, W., Vosniadou, S. y Carretero, M. (eds.). New trends on conceptual change. Londres: Elsevier.

POZO, J.I., SCHEUER, N., PÉREZ ECHEVERRÍA, M.P. y MATEOS, M. (1999). El cambio de las concepciones de los profesores sobre el aprendizaje, en Sánchez, J.M. et al (eds.). Educación científica. Madrid: Ediciones de la Universidad de Alcalá.

REDER, L.M. (ed.) (1996). Implicit memory and metacognition Hillsdale, N.J.: Erlbaum.

RODRIGO, M.J. (1997) Del escenario sociocultural al constructivismo episódico: un viaje al conocimiento escolar de la mano de las teorías implícitas, en Rodrigo, M.J. y
Arnay, J. (eds.). La construcción del conocimiento escolar. Barcelona: Paidós.

RODRIGO, M.J. y CORREA, N. (1999). Teorías implícitas, modelos mentales y cambio educativo, en Pozo, J.I. y Monereo, C. (eds.). El aprendizaje estratégico: enseñar a aprender desde el currículo. Madrid: Santillana.

SÁNCHEZ, E. (1999). Texto y conversación: de cómo ayudar al lector a conversar con los textos, en Pozo, J.I. y Monereo, C. (eds.). El aprendizaje estratégico: enseñar a aprender desde el currículo. Madrid: Santillana.

SANMARTÍ, N., JORBA, J. e IBÁÑEZ, V. (1999). Aprender a regular y a autorregularse, en Pozo, J.I. y Monereo, C. (eds.). El aprendizaje estratégico: enseñar a aprender desde el currículo. Madrid: Santillana.

SCHRAW, G. y MOSHMAN, D. (1995). Metacognitive theories. Educational Psychology Review, 7(4), pp. 351-371.

VOSNIADOU, S. (1994) Capturing and modelling the process of conceptual change. Learning and Instruction, 4(1), pp. 45-69.

WAGENSBERG, J. (1993). Sobre la transmisión del conocimiento científico y otras pedagogías. Substratum, 1(2), pp. 87-95.

WHITE, R. (1994). Conceptual and conceptional change. Learning and Instruction, 4(1), pp. 117-121.

WHITE, R. (1999). Condiciones para un aprendizaje de calidad en la enseñanza de las ciencias. Enseñanza de las Ciencias, 17(1), pp. 3-15. 